\title{
Information Empowerment Through Mobile Learning
}

\author{
Raymond Koon Chuan Koh \\ National University of Singapore \\ IDMI, 21, Heng Mui Keng Terrace, \\ Singapore 119613 \\ (65) 65165951
}

raymondkoh@nus.edu.sg

\author{
Henry Xin Liong Tan \\ National University of Singapore \\ IDMI, 21, Heng Mui Keng Terrace, \\ Singapore 119613
}

(65) 65165951

xinliong@gmail.com

\author{
Henry Been-Lirn Duh \\ National University of Singapore \\ IDMI, 21, Heng Mui Keng Terrace, \\ Singapore 119613
}

(65) 65165951

ecedbl@nus.edu.sg

\begin{abstract}
Information empowers those who make sense from its successful interpretation; this is especially true on the subject of weather where the recipient is required to have prior forms of fundamental understanding towards the natural occurring phenomenon and its effects. This study facilitates the learning of complex weather phenomena for children using a unique educational approach using mobile technology. The initiative runs under Singapore's National Weather Study Project (NWSP), which already operates a network of mini weather stations in schools across the state to promote environmental awareness and support dynamic interactions with real time data. Its data is accessible via Web, Microsoft SensorMap and Google Earth interfaces. IWIS, an application for the recent Google Android mobile platform taps onto the fusion of mapping and the weather project server's data to present to children accessible local multi-variate weather data depicted using visual metaphors and propagates the development of mobile learning applications in the imminent future.
\end{abstract}

\section{Categories and Subject Descriptors}

H.5.1 [Information Interfaces and Presentation]: Multimedia Information Systems - Evaluation/methodology, Hypertext navigation and maps.

\section{General Terms}

Documentation, Information Design, Experimentation, Human Factors, Languages

\section{Keywords}

Mobile learning, information visualization, visual cognition, knowledge management

\section{INTRODUCTION}

Weather information consists of a large array of raw data ranging from the common temperatures (current/forecasted), rainfall levels and wind directions to the more abstract forms as such as humidity, atmospheric pressure and tide levels, usually charted against a date-time graph. This may be useful only as an archival repository for weather data as it does not inform how any fluctuation in it has any interrelations to other weather phenomenon exhibited over the same period [1]. The detailed information range may be beneficial to a researcher to map out the different relationships that may be exist in the multi-variate data model [1], but to the "non-informed", it may appear unfamiliar. This knowledge however is important today, as it is highly relevant and applicable to many practical aspects of our Copyright is held by the author/owner(s). MobileHCI'09, September 15 - 18, 2009, Bonn, Germany. ACM 978-1-60558-281-8. lifestyles and activities, regardless of our social differences.

Visualization of information has been a popular research field even though it is fairly new. Its goal is mainly to perceptualize information. The focus of this research hence is to address this avenue of information conveyance to children by amplifying cognition by developing effective visual metaphors for directly mapping abstract data [2]. Apart from exploring multi-axial plotting means to represent temporal data, the interaction experience is enhanced using the interdisciplinary course of design and arts where various subjective artistic expressions can be used to link the multi-faceted information and provide a direct visual interpretation of the data. This form of emerging work is also known as visualization art and it aims to provide a channel of visualization through the use of artistically inclined but data driven form of representation.

\section{MOBILE TECHNOLOGY 2.1 Embracing mobility}

The advancements in mobile technology come as an opportune door to expedite this 'weather information understanding' effort by exploiting current visualization methodologies. Recent modern features (i.e. increasing computing power, application tool support, high-speed Internet connectivity, color-rich resolutions etc), exorbitant worldwide user penetration rates and dynamic ubiquitous nature of mobile phones present the technology as an ideal multimedia enriched hub for learning, analysis and information dissemination on demand, apart from being simply a communication tool [3]. Coupled with the common Global Positioning System (GPS) function, children will be able to explore and enjoy geographically relevant location-based services while experiencing this new learning approach.

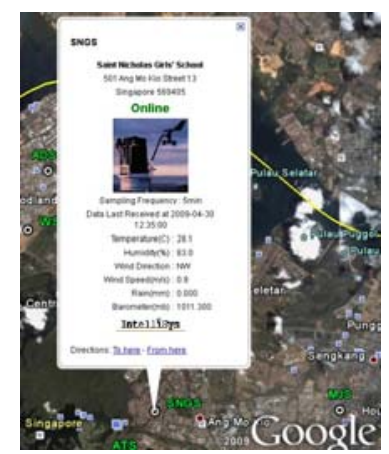

Figure 1. Weather data viewed in Google Earth 


\subsection{IWIS}

Considering some of the design principles proposed by [4], "Interactive Weather Information System" or IWIS is developed as a prototype mobile application using unique design-oriented visual metaphors to represent a select group of weather information that is retrieved from the weather stations' database (Figure 1) to be presented to children (Figure 2).

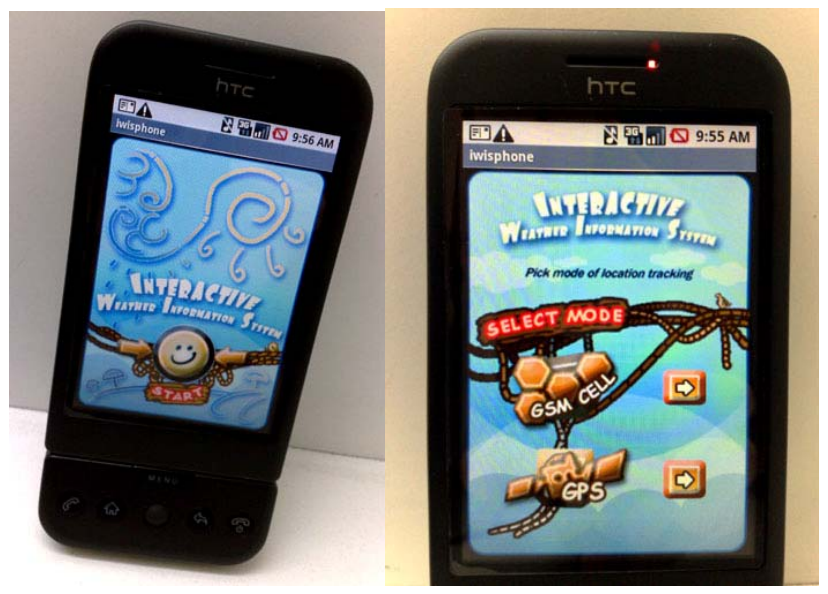

Figure 2. IWIS on Google Android in HTC's Dream mobile

\subsection{Location-based tracking}

IWIS is 'location-aware' by offering two modes of position tracking (Cell ID or GPS) to the user (Figure 2). The project's weather stations are depicted on Google Mobile Map's interface in the mobile device, giving a clear spatial representation of the stations' physical locations relative to the user's present geographical coordinates (Figure 3) with the latest weather information available on demand (by tapping a station's marker on map) [5].
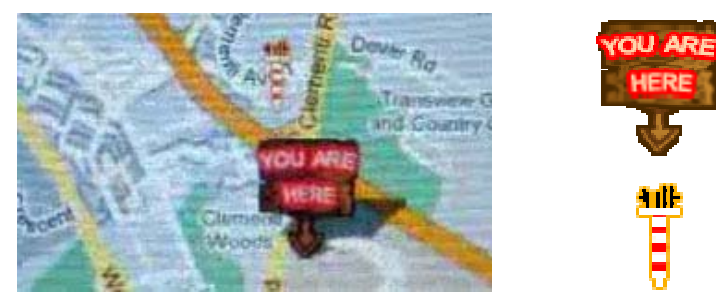

Figure 3. IWIS/Google Map integration using markers for user's and a NWSP mini weather station's locations.

\subsection{Visual metaphors}

Shapes (2D and 3D), sizes and colors metaphors (Figure 4) are collectively used to visually represent real-time weather data in IWIS (Figure 5 Left). One important function of such utilization is that it establishes and supports the possible linkage of familiar concepts to unknown ones [6]. Hence, the depth of a metaphor's visualization coressponds to the ease of conveying the intended meaning.

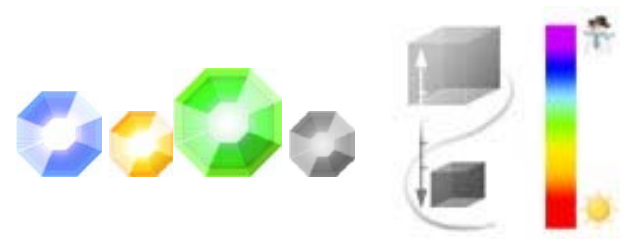

Figure 4. Visual metaphors in IWIS

In addition, the full scalar data from the selected weather station can be still available for viewing. In IWIS, we filtered this down to a selected (but easily re-configurable) range of data types that we would like to present on that screen (Figure 5 Right).
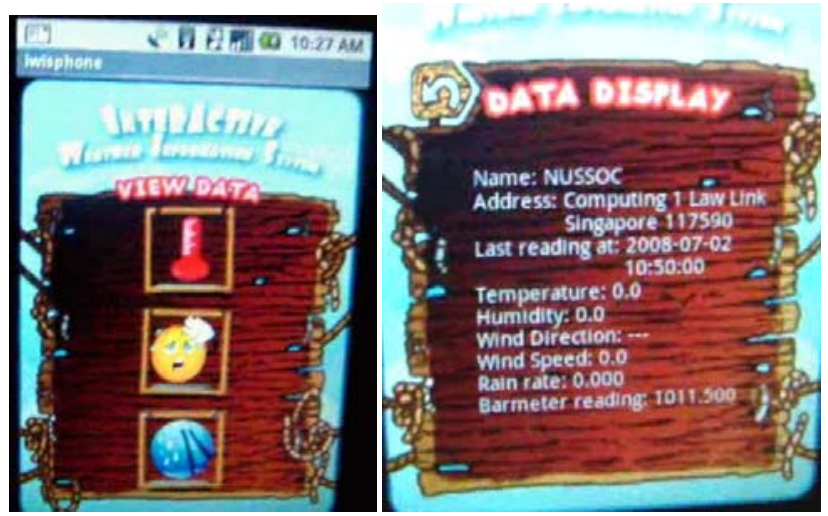

Figure 5. Left: Weather data retrieval options in IWIS prototype. Right: Weather data linkage test in IWIS (Google Android)

\section{$2.53 \mathrm{D}$ objects}

A 3D cube is used as part of the information visualization in IWIS. It features the following attributes for translating information:

\subsubsection{Opacity}

Opacity values relate how recent downloaded data from the weather stations are. An opaque appearance (of the 3D cube) would mean that the data is up to the current date of retrieval while a translucent look will mean that the weather data is more than a day old. In this case, it represents the linkage as if it is a surrealistic fading away of information through time.

\subsubsection{Size}

Magnitude of the weather data is depicted in IWIS by the size of the object in a proportional scale that is developed for this purpose.

\subsubsection{Shading}

The cube's shading is used to represent the intensity of a specific condition (i.e. solar radiation) of the weather phenomenon. 

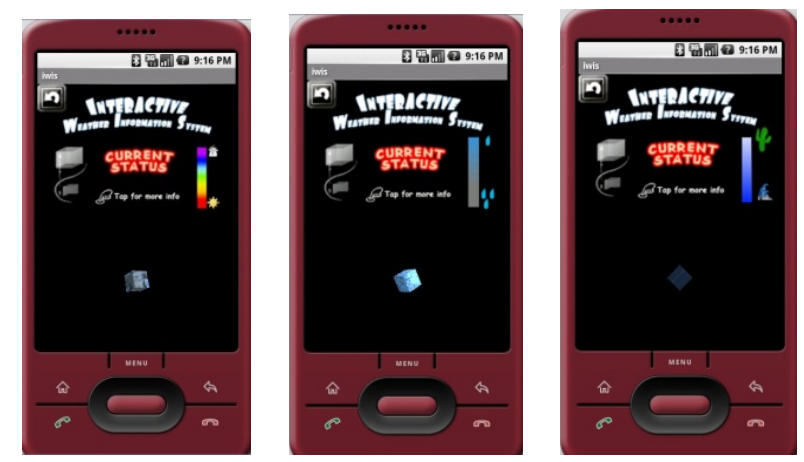

Figure 7. Retrieved weather data in the respective order of temperature, humdity and rainfall

\subsubsection{Texture Overlay on 3D Cube}

Textures can communicate a ubiquitous visual sense. IWIS aims to provide information at the ease of a glance by mapping selective textures directly onto the 3D cube (Figure 7), conveying the meaningful representations of the associated but varied weather attributes (Figure 8).
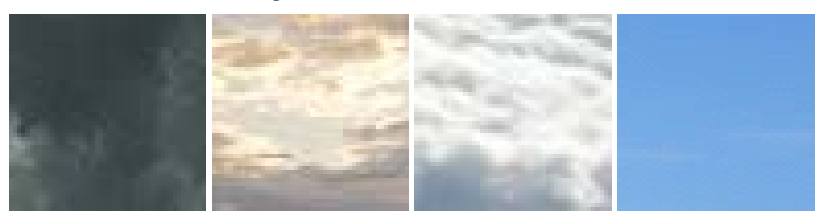

Figure 8. Bitmap images are used as textures to represent the humidity of the current weather condition. From left, thunderstorm, light shower, cloudy and clear weather conditions are depicted

\subsubsection{Visualization Art}

Information visualization is not limited to only charted or graphed values in order to find the hidden patterns and relationships between different data sets. IWIS exploits the use of creatively designed metaphoric icons to represent intertwined weather data, allowing one to better understand the cross-linked information associated with the icons.

\subsubsection{Color Coding}

Colors have been the most consistent form of representation tool as humans tend to associate certain colors to specific types of emotions or moods. In IWIS, this color association is intentionally set up to define the sets of climate. The different shades of a color are also used in presenting the different magnitudes of the data sets.

\section{CONCLUSION}

IWIS exploits mobile technology's interactive visual and location capabilities to appeal and append a meaningful explanatory essence to abstract topics in weather, thereby empowering meaningful dynamic information retention to new audiences who have no prior knowledge on its specifics to be able to form meaningful interpretation through its unique visualization technique. It is also able to overcome the display constraints to hold large amounts of information on the space-limited screens of mobile devices.

This portable approach can be beneficial in facilitating the learning of interrelated subjects beyond the classroom. Future work will seek to explore the dynamic informational links to historical weather data and also to explore the cognitive effects of such visual representations to specific educational information so as to maximize the design's communication efficiency to children.

\section{ACKNOWLEDGMENTS}

This initiative is supported by the Singapore National Research Foundation (NRF-20008-IDM-001-MOE-016) and the National University of Singapore (R 263-000-488-112).

\section{REFERENCES}

[1] Nocke, T., Flechsig, M., and Böhm, U. (2007). Visual exploration and evaluation of climate-related simulation data. In Proceedings of the 39th Conference on Winter Simulation: 40 Years! the Best Is Yet To Come. 2007.pp.703-711.

[2] Lau, A., and Moere, A.V. (2007). "Towards a Model of Information Aesthetics in Information Visualization". 11th International Conference Information Visualization. 2007. pp.87-92.

[3] Pombinho, P., Paula, A., Maria, A., Carmo, B. 2007. Georeference Information Visualization on Mobile Devices, 1749-016.

[4] Reichenbacher, T. (2004). Mobile cartography-adaptive visualisation of geographic information on mobile devices, PhD Thesis, Technischen Universitat Munchen, 2004.

[5] Nivala, A-M. and Sarjakoski, L.T. (2005). Adapting map symbols for mobile users. In Proc. of the 22nd International Cartographic Conference, July 9-16, A Coruna, Spain, 2005.

[6] Mountford, S.J., "Tools and Techniques for Creative Design”. In The Art of Human-Computer Inte ceDesigr B. Laurel Ed., Addison Wesley, 17-30, (1990) 